\title{
Preparing Inclusive Class for the Children with Special Needs during COVID -19 Crisis
}

\author{
Nisha Valvi, Sanjeev Sonawane and Priti Jadhav"* \\ Department of Education and Extension, Savitribai Phule Pune University, Pune, India \\ *Corresponding author: gourinisha77@gmail.com
}

Received: 17-09-2020 Revised: 19-11-2020 Accepted: 09-12-2020

\begin{abstract}
Ongoing COVID-19 pandemic is served as an opportunity to re-think how emergency education planning can be inclusive of children with disabilities and the need for accessible and inclusive education. As the COVID-19 pandemic continues to spread across the globe, schools are closed in 180 countries, leaving an estimated 1.5 billion children out of school. In addition, $80 \%$ of persons with disabilities live in developing countries where access to education is an ongoing challenge. The impact of COVID-19 is likely to be worse for people in lower socio-economic groups, and children with disabilities face an even greater risk of being left behind. In this paper some challenges, ways and strategies are discussed so that we can move the Post COVID-19 agenda forward to make education truly disability inclusive and easier.
\end{abstract}

Keywords: Inclusive class, COVID -19 crisis, Children with special needs.

Online learning has been particularly difficult for students with disabilities. Online classes have proved to be pointless for children with mild learning difficulties, such as attention deficit hyperactivity disorder, may struggle with independent work in front of a computer. Online teaching has been welcomed by both educators and students as an opportunity to get acquainted with the technologically led advanced learning system like that of the western countries. However, e-teaching when put before those who are not much aware about the virtual system of education, is futile. People with disabilities mostly fall under the category of vulnerable groups. In this pandemic students with disabilities face many problems related to accessibility and learning resources. In India, major population resides in remote areas having less connectivity to internet. To talk about inclusion of persons with disabilities in higher education institutions, it is important to know that the pandemic affects such people differently and makes them more vulnerable in comparison to others.
So, what can we think about the inclusion of students with disabilities? There are expected challenges that are faced by these students. Firstly, shifting classes from face-to-face interaction to online mode is daunting for those who have visual and hearing impairment. Majority of the students lack internet connectivity and laptops for online classes. Even if they have the required gadgets, it's difficult for them to use online learning applications. Second challenge faced by disabled students is related to evaluation and assessment. It would be a complex procedure for students with disabilities to understand assignments given online in general as there is no expectation of its significance with respect to the level and category of disability. Moreover, the integrity of honesty in giving online feedback will be a challenge that teachers are going to face while they are in evaluation. The third reason is related

\footnotetext{
How to cite this article: Valvi, N., Sonawane, S. and Jadhav, P. (2020) Preparing Inclusive Class for the Children with Special Needs during COVID -19 Crisis. Educational Quest: An Int. J. Edu. Appl. Soc. Sci. 11(4): 183-187.
}

Source of Support: None; Conflict of Interest: None 
to the unavailability of resources for making an arrangement of online equipment's, as majority of the students with disabilities are living below the poverty line in India. They are dependent on the lectures delivered in the classroom and can't buy laptops, smartphones, internet connectivity on their own. Fourth challenge that needs to be addressed is related to the mental health of students. As disabled students face multiple psychological problems due to negative attitude of the society and system, they are more prone to getting depressed due to lack of facilities to deal with this situation of uncertainty.

Keeping the above challenges in mind, the authorities must take it seriously and come up with possible solutions of the issues responsible for the exclusion of students with disabilities. If the agenda of online classes is 'no compromise on equality of education', then we must look carefully that no one should left behind in this great hour of need. Along with the promising equality of education, the allocation of resources and training of online learning of all students must be a priority of both administration and government. Thus, in order to facilitate students with disabilities, all schools need to setup a special task force who with time identify the potential needs of students and thereby design clear directions for faculty members in taking online classes, midterm exams, assignments and projects.

\section{Ways and strategies to prepare inclusive class in COVID 19 crisis}

The disable students are at a higher risk for contracting COVID -19 with reduced access to social distancing, health care services, personal protective equipment such as masks, basic hygiene facilities and sanitation. Many of these students with physical and mental disabilities may not be able to wash their hands not only because of a lack of running water and soap, inaccessibility to clean water but because they are not able to turn on a faucet and wash their hands due to their physical deformities. This is more so when disabled students are wheelchair bound and are victims of Polio and Hansen's disease which may leave them crippled and unable to perform simple tasks without assistance. (http:// www.ipsnews.net/2020/08/covid-crisis-challengespeople-disabilities-hansens-disease/)

\section{Collaborative approaches}

A collaborative team approach is typically adopted when children need special education services, with students, parents, teachers, educational assistants and other school and community-based staff working together to plan and implement programming. The human supports required by many students with disabilities in order to flourish socially, emotionally and academically, it's a challenge for systems to provide the right types and amounts of distance learning opportunities and supports.

Some students who find structured schooling to be a poor fit are benefiting from the shift to a more relaxed pace and self-directed learning. They can dance, jump and wiggle to their heart's content. They can engage in tasks for a length of time that works for them and make choices during their days. The right kind of distance learning paired with accessible technology and available supports may be a great fit for them.

Other students who thrive on a predictable routine, struggle with transitions and depend on the strong relationships built over time with teachers and educational assistants are experiencing a range of emotions, including worry, fear, anger and sadness. Some feel that they've lost a second home and may not understand why. Some students may also communicate these difficult emotions in ways that are challenging for parents, caregivers and siblings.

\section{Family challenges}

Parents of students who have worked hard to make gains in their learning worry that they may fall behind. This is particularly the case where parents may not have the English or French language skills to provide help or the time to engage with their children because of work and life commitments. Where internet access and technology in homes is the exception rather than the rule, the situation is further complicated. Parents who typically work in partnership with school staff and community organizations are especially struggling with the disappearance of face-to-face networks and collaboration in supporting children.

\section{Social, emotional supports}

It's helpful for all families, and necessary for some, 
to find ways of connecting with others for both parents and students. Connecting with school staff, community groups, family and friends may look different right now, but some of the benefits remain. We should need to emphasize social-emotional learning alongside academics as key to well-being. This is vital right now and will continue to be in the tricky transitions ahead. Across the country, school staff have reached out to disabled students in different ways. Some are recording themselves reading favourite books and others are connecting by phone or video chats. Some regions have social workers or psychologists reaching out to students or discussing mental health via virtual classes.

\section{Peer connections matter}

Connections with peers are also crucial for students. Building and maintaining friendships is not always easy for students with disabilities. Many students rely on school-based networks for friendship and may not have these connections in their neighbourhoods, particularly if they haven't been in an inclusive environment at school. Some community groups have come up with creative solutions to virtually connect students where students with all types of disabilities can chat, play games, create art work and take part in talent shows. Peer and social support for parents is also crucial at this time connecting with others who are in similar circumstances, seeking respite supports and collaborating with schools where possible.

\section{Expanding inclusion}

Our conversations with parents are bringing to light issues of inclusion and exclusion, of balancing academic and social needs and development and of the challenges of differentiating distance learning in ways that allow it to be meaningful.

It's our hope that whatever schooling looks like in the fall in context of coronavirus, our schools and communities can create learning opportunities that reflect the unique strengths and needs of all our students.

We will be continuing to encourage educators and school communities to imagine how can we develop social networks among students with and without disabilities, how can we build authentic communities that live inside and outside the brick and mortar of a school and how can we support partnerships between families and school staff. (https://theconversation.com/coronavirus-distancelearning-poses-challenges-for-some-families-ofchildren-with-disabilities-136696)

\section{Strategies to overcome a challenge during COVID 19 of inclusive setting}

Irrespective of being a parent or a teacher, it is important to keep things in perspective and look at the bigger picture. Remember, we are not trying to 'cure' learning disabilities, instead we must look at providing social, emotional and academic skills that will help them overcome their challenge and make them more resilient. Give your children the emotional and moral support they need during this time, especially as they navigate to the new territories of virtual learning. Research on learning disability programmes, therapies and educational techniques.

\section{Focus on child's strengths}

Don't let a child be defined and confined by their learning disability. This is just one area of weakness. Instead, encourage and pay attention to their many strengths and unique talents. It is important that you continue to allot time for creative activities too especially during this lockdown. Online teaching has opened a whole new world of opportunities for us to learn various skills.

\section{Take charge of your role as educator}

As most parents double up as teachers during this time, it is important to identify how your child learns best. A short conversation with a special educator will give you a clear idea on whether your child is a visual, auditory or kinaesthetic learner. There are multiple resources available online to assist you with various strategies, tips and tools to help your child learn the way they can. Recognise that schools are only part of the solution but your support, interest and encouragement can have a greater impact on their lives.

\section{Lifestyle and habits}

This pandemic has made us contemplate on our lifestyle, habits and choices. A rested and well looked after mind and body is key; adequate sleep, exercise and nutrition will help with better focus and concentration. Children with learning 
disabilities benefit the most from routines so make sure they have one before and after virtual school. Create a study space for them and teach them how to organise and maintain the classwork so you don't have to do it for them.

\section{Seeking professional help}

Recognising a build-up of frustration, anxiety or stress in both parent and child is important. Communication with teachers, special educators or therapist about the struggles that are facing by child for better assistance. Reach out to a counsellor or psychologist. Talking about frustrations with a professional who can suggest healthy ways to cope with the situation will help parent and child to deal with stressful situations with more compassion and empathy.

\section{Role of teachers and parents in COVID 19}

Accept the situation: Children with intellectual disabilities are more likely to make sense of the pandemic and associated crises from the perspective of their own physical and emotional safety. Family members need to understand and accept that expecting these children to put their family's needs before their own is unrealistic.

Use of Assessment tools: Through various types of assessments like Oral exams, Storytelling, Group activities, MCQ's, Quiz,Demonstration knowledge, Video clips, Hobbies, Nature picture art, Role model, Phonetic art a teacher can assess child's progress.

Set a routine: Even though your regular routine with your child is compromised, maintaining a daily routine is important for your child and you as well. Use visual aids to set a new routine with your child and other members of the family, just so you're prepared enough to respond to the child's needs as well as any crisis.

Use visual aids: Prepare everybody's routine on a plain sheet of paper with pictures and bold letters. These pictures will work as reminders for your child and other family members as well. You can also use this method to explain the pandemic, lockdown and guidelines in simple steps.

Observe: Learn to take cues from your child to understand what indoor activities they enjoy the most and which ones annoy or scare them. Set aside time to bond over all the games and activities your child enjoys.

Be friendly: While it's natural for most parents to engage with the child to give instructions or guide them through activities, it's equally important for the child to see you as a friendly presence. Engage in the child's play and rituals to ensure this.

Reduce demands: Stay alert to the number of demands you place on your child. Asking them to wash their hands, study, take a bath, etc is important, but try putting these in other formats instead of just demanding them, because that can lead to resistance, conflict or passive following.

Select your goals: Set some teaching goals every week with the help of your specialist, so that your child can continue to learn. However, be realistic about these goals and also focus on daily life skills which are easy and can be a part of your child's routine. (https://www.firstpost.com/health/10-waysyou-can-help-children-with-intellectual-disabilitiesduring-the-covid-19-pandemic-8396761.html)

Don't push excessively: Children with intellectual disabilities are likely to have difficulty in coping with academic tasks, so you mustn't push the child excessively. This can reduce your child's motivation and create an aversion too. Consult your specialist to create realistic academic goals.

Stay in touch: Maintain regular contact with your child's specialist and other members of your regular team of professionals like occupational therapists, special education teachers, speech and language pathologists, psychologists, etc. Coordinate with them to ensure that your child is on track and doing well despite the pandemic and lockdowns.(https:// indianexpress.com/article/lifestyle/life-style/how-tosupport-children-special-needs-6470363/)

\section{CONCLUSION}

All the teacher, parent and psychologists, stallholders need to do is work together for betterment of disabled student in this pandemic. Communication, timely discussion, counselling, use of technology or a special software, project-based learning, daily life activities, holistic approach towards child's learning, different assessment tools, comprehensive support are some strategies can be used to support inclusive learning. 


\section{REFERENCES}

https://www.globalpartnership.org/blog/inclusive-responsecovid-19-education-children-disabilities

https://news.careers360.com/covid-19-learning-strategiesadopted-are-not-inclusive-says-report

https://countercurrents.org/2020/04/covid-19-and-onlineteaching-challenges-for-the-inclusion-of-disabledstudents-in-higher-education/

https://theconversation.com/coronavirus-distance-learningposes-challenges-for-some-families-of-children-withdisabilities-136696 https://indianexpress.com/article/lifestyle/life-style/how-tosupport-children-special-needs-6470363/

https://theconversation.com/how-coronavirus-highlightsthe-everyday-challenges-of-people-with-learningdisabilities-139518

https://www.firstpost.com/health/10-ways-you-can-helpchildren-with-intellectual-disabilities-during-the-covid19-pandemic-8396761.html 
